\title{
Acute patellofemoral instability in children and adolescents
}

\author{
PIERLUIGI ANTINOLFI ${ }^{1}$, MATTEO BARTOLI ${ }^{2}$, GIACOMO PLACELLA ${ }^{2}$, ANDREA SPEZIALI $^{3}$, \\ VALERIO PACE ${ }^{4}$, MARCO DELCOGLIANO $^{5}$, CLAUDIO MAZZOLA $^{6}$ \\ ${ }^{1}$ Department of Orthopaedics, Università di Perugia, Italy \\ ${ }^{2}$ Department of Orthopaedics, Università Cattolica del Sacro Cuore, Policlinico Gemelli, Rome, Italy \\ ${ }^{3}$ Department of Orthopaedics, Ospedale di Orvieto, Italy \\ ${ }^{4}$ Royal National Orthopaedic Hospital, Stanmore, London, UK \\ 5 Ospedale Nazionale di Lugano Civico e Italiano, Lugano, Switzerland \\ ${ }^{6}$ Department of Orthopaedics, Ospedali Galliera di Genova, Italy
}

\begin{abstract}
Patellofemoral problems are considered to be among the most frequent causes of knee pain in children and adolescents. Correcting bone abnormalities through specific and targeted interventions is mandatory in skeletally immature patients. Medial patellofemoral ligament (MPFL) reconstruction is the preferred procedure, but there are several important precautionary considerations that the surgeon must take into account. It must always be remembered that MPFL rupture is the result, not the cause, of an altered extensor mechanism; therefore, patellar stabilization with MPFL reconstruction is only the first step to be accomplished in the management of an MPFL rupture. If other anatomical alterations are encountered, alternative/additional surgical procedures should be considered. If MPFL rupture occurs without associated anatomical or functional knee alterations, an appropriate rehabilitation program after MPFL reconstruction should be sufficient to achieve a good outcome.

In conclusion, an acute patellar dislocation should be managed conservatively unless there is evidence of osteochondral damage or medial retinaculum lesions. Osseous procedures are contraindicated in children, while MPFL anatomical reconstruction with "physeal sparing" is the primary surgical option.
\end{abstract}

\section{Corresponding Author:}

Matteo Bartoli, MD

Department of Orthopaedics, Università Cattolica del

Sacro Cuore, Policlinico Gemelli

L.go Agostino Gemelli 8, 00168 Rome, Italy

E-mail: matteobartoli1@gmail.com
Keywords: pediatric, medial patellofemoral ligament, patellofemoral, instability, physeal sparing.

\section{Introduction}

Patellofemoral problems are considered to be among the most frequent causes of knee pain in children and adolescents (1), with incidence rate, for 10-18 year olds, of about 29 per 100,000 people per year (2).

Patellofemoral malalignment is defined as abnormal patellar tracking with lateral displacement or lateral tilt of the patella, or both (3). Some use this term to indicate malpositioning of the knee between the body and the foot, with a consequential impact on the patellofemoral biomechanics.

Patellar instability is a non-specific term that includes several specific diagnoses: each type of instability has a specific functional or anatomical cause and should therefore be treated according to its etiology (4).

In this setting, we should consider:

- patellofemoral syndrome as pain occurring during extension of the knee;

- patellar maltracking as a dynamic incongruence between the patella and the trochlea;

- patellar malalignment as an anatomical incongruence between the patella and the trochlea;

- dysplasia as a growth abnormality.

The annual incidence rate of first-time (primary) patellar dislocations in children ( $<14$ years) is $43 / 100,000$ (5).

In a study of pediatric sports-related injuries, the percentage of female patients with patellofemoral pain was approximately three times greater than the per- 
Table 1. Predisposing factors to patellar dislocation.

\begin{tabular}{ll}
\hline MAJOR FACTORS & SECONDARY FACTORS \\
\hline Trochlear dysplasia & Patellar shape \\
\hline Patella alta & Valgus knee \\
\hline Increased TT-TG distance & Genu recurvatum \\
\hline Lateral patellar tilt & VMO deficiency \\
& Generalized hyperlaxity \\
& Q angle \\
& Hypoplastic lateral femoral condyle \\
& Internal femoral torsion \\
& External tibial torsion \\
\hline
\end{tabular}

Abbreviations: TT-TG = tibial tuberosity-trochlear groove; $\mathrm{VMO}=$ vastus medialis obliquus muscle.

centage of male patients (14.3 vs $4.0 \%$, respectively; $\mathrm{p}$ $<$.001) (6).

At the current state of the literature, the main risk factors for patellar instability, clearly highlighted (Tab. 1), are: trochlear dysplasia, patella alta, lateralized tibial tubercle with increased tibial tuberosity-trochlear groove (TT-TG) distance, and lateral patellar tilt (7).

There is a high incidence of patellofemoral dysplasia in patients with acute patellar dislocation; the Wiberg and Baumgart classifications are still valid and show how the shape of the patella is also a key element to be taken into account when diagnosing and managing patella disease (8).

There is a statistically significant clinical association between the shape of the patella and patellar tilt: the more the patella tilts, the more it appears to be dysplastic (9). The extent of patellar tilt is correlated with static and dynamic factors: if the quadriceps is relaxed, the tilt depends on the morphology of the bone surfaces, but if the quadriceps is contracted it depends on the balance between the medial component and the lateral component of the muscle itself.

In patients with patellofemoral instability, increased tilting, measured in dynamic phase, correlates directly with vastus medialis obliquus (VMO) muscle dysplasia. In these cases the VMO does not distally insert into the upper third of the patella but inserts directly on the quadriceps tendon, losing its function as a restraint to lateral translation of the patella and favoring an increase in lateral patellar tilt. VMO dysplasia is a congenital disease; in affected individuals the patella becomes aligned with the vastus lateralis, but not with the VMO, and this leads to other anatomical and biomechanical changes in the lower limb, increasing the tendency of the patella to dislocate. The presence of a patellar deformity does not seem to influence other major risk factors for lower limb abnormalities. However, the various types of soft tissue dysplasia are found more frequently in patients with patellar dislocation than in the general population.

Dysplasia of the femoral groove is the main anatomical abnormality in patients with a predisposition to lateral patellar dislocation. This condition affects the function of all the other patellar stabilizers as it increases the chances of them failing to stabilize the patella in its anatomical position. Knee joint laxity, which may also reduce the ability of the medial retinaculum to prevent lateral patellar dislocation, is also closely related to this pathological condition.

It has been shown in the literature that the presence of just one of these factors can independently lead to dislocation; it is also well known that their coexistence may exponentially increase the risk of patellar dislocation (7).

The presence of both trochlear dysplasia and patella alta, decreasing the capacity of the patella to lie within the femoral groove at any angle of knee flexion, directly contributes to a greater risk of acute and recurrent patellar dislocation, as the anatomical lateral support normally provided by the trochlea is reduced. Anatomically, the trochlea has a concave shape; a characteristic of the trochlea is that it is morphologically similar to the overlying cartilage $(10,11)$. Trochlear dysplasia is defined as a flat proximal articular surface 
separated from the distal joint surface by just a small intra-articular space; occasionally the trochlea has a convex shape, in which case the cartilage is thinner in the center of the trochlea and thicker at its sides. Inadequate depth of the femoral groove can affect both the morphology and the function of the entire knee joint; alternatively the problem can be localized to a specific area of the groove and surrounding anatomical structures, as occurs when the dysplastic process involves only the highest aspect of the femoral groove. A satisfactory classification of trochlear dysplasia was proposed by Dejour et al. (12).

If the groove is flat, the patella is unable to lodge in it during knee flexion, especially in early degrees of flexion. In the presence of this deformity the stress forces are distributed mainly on the lateral facets, leading to arthritic changes in the long term.

Patella alta is also known to be associated with a high dislocation rate; in most cases the etiology is idiopathic. Axial deformity and rotational changes in all the skeletal segments of the lower limb may play a crucial role in patellar instability: increased femoral anteversion or tibial torsion may disrupt patellofemoral tracking (13-15). Powers et al., in 2003, using dynamic acquired MR images in patients with patellar instability, showed patellar tilt and dislocation (as from $10^{\circ}$ of extension) to be due mainly to femoral anteversion rather than other abnormal patellar tracking (16). External tibial torsion influences the quadriceps angle ( $\mathrm{Q}$ angle), which differs significantly in patients affected by patellar instability compared with the standard population; this mechanism results in the creation of a lever arm that impedes the action of the quadriceps, increasing lateral patellar instability. A valgus knee may also alter the $\mathrm{Q}$ angle and can potentially be responsible for patellar instability, especially if it is associated with abnormal external rotation of the tibia. This is particularly true during quadriceps contraction in the last degrees of extension. Furthermore, this clinical scenario may easily lead to distension of the medial aspect of the joint capsule, increasing the laxity of the knee. A valgus knee is not only the result of bone abnormalities, but can also be a consequence of muscle imbalance, muscle weakness or abnormality of the ipsilateral hip, knee or ankle.

Correcting the above-mentioned bone abnormalities through specific and targeted interventions is manda- tory in skeletally immature patients; all abnormalities should be specifically corrected $(17,18)$.

\section{Treatment of acute dislocation}

There is enormous debate over the most appropriate treatment of acute dislocations. The first step in their management is usually conservative treatment with the use of a brace, off load walking, stretching and muscle strengthening exercises. For many years considerable attention has been paid to strengthening of the quadriceps muscle, especially the VMO and rectus femoris, although the lateral retinaculum, hamstrings, quadriceps, Achilles tendon and iliotibial band should also be taken into account. Some Authors have drawn attention to the therapeutic importance of stretching and complete muscle re-education, highlighting the role played by correct contraction of the quadriceps and adductor muscles in dynamizing the medial patellofemoral ligament (MPFL), which drives the patella within the trochlea.

The conservative approach is a widely chosen management option, unless there is evidence of osteochondral damage or anatomical deficiencies (19).

Literature shows that a lesion in the MPFL-VMO overlap region has a greater chance of healing well when it is located close to the patellar insertion, whereas a lesion close to the femoral insertion will not easily heal on its own; furthermore, conservative treatment has a $52 \%$ failure rate, in terms of redislocation (20).

Surgical management of first patellar dislocation is associated with a significantly lower risk of redislocation $(15 \%)$ compared with non-surgical management, and with a significantly higher risk of patellofemoral osteoarthritis $(21,22)$.

The incidence of patellofemoral osteoarthritis may be related to the surgical techniques chosen: distal realignment, muscle-tendon transfers, lateral release and medial reefing are not effective and not really safe (23). When an adolescent requires surgical treatment, osseous surgery is contraindicated as it is not safe; medial reefing has poor results, while lateral release does not improve functionality but could improve chondromalacia $(23,24)$.

It is well known that the MPFL is the most important static patellofemoral joint stabilizer and that its rupture 
is unavoidable in cases of lateral dislocation: the maximum elongation it can undergo is $20-30 \%$, therefore $18-20 \mathrm{~mm}$ at most, which is far less than the entire width of the patella which often exceeds $40 \mathrm{~mm}$ (25). MPFL reconstruction should therefore be the primary surgical option in adults (25). Indeed, untreated MPFL rupture can cause various anatomical and functional problems which lead to recurrence of the dislocation and abnormal growth of the joint.

MPFL reconstruction should also be the preferred procedure in skeletally immature patients, in whom there are several important precautionary considerations that need to be taken into account: drilling procedures must not be performed close to the growth plate; the patella is smaller in adolescents than in adults; excessive medial tension and tunnel malpositioning must be avoided.

Shah et al., in 2012 (26), demonstrated that periosteal sutures, compared with other techniques, have a higher rate of positive apprehension test, recurrent dislocation and hyperlaxity; minor adverse events are more frequent when using the patellar bone tunnel technique. They also found that graft fixation performed at $>60^{\circ}$ of knee flexion is the safest technique when considering major complications: it is important to achieve good correction of the instability with no risk of excessive medial pressure.

Even minimal technical errors during the surgical procedure might lead to significant increased stress on the patellofemoral joint (27). Patellar fractures, in tunnelling techniques, are considered to be the worst possible complications; they occur in $0.4 \%$ of cases (27).

Another critical aspect is the presence decreased maximum knee extension, observed in $13.4 \%$ of patients (26); this seems to be due to excessive graft tension or incorrect femoral fixation.

The surgeon must take particular care when operating close to the physis in adolescents, for two important reasons: to avoid patella alta and growth disorder. Indeed, it is well known that the femoral physis lengthens distally during growth, and if the femoral insertion of the MPFL is placed proximal to the physis the patient could develop a patella alta at the end of childhood. To avoid this complication the MPFL femoral insertion should be always be positioned distally to the growth plate, even though the native femoral MPFL insertion is located $4 \mathrm{~mm}$ (on average) proximal to the femoral distal growth plate.
To avoid growth disorder, the femoral tunnel should be drilled at very low speed and at a distance of at least $8 \mathrm{~mm}$ from the femoral physis in order to prevent harmful effects of high temperature on the growth plate.

Using fluoroscopic imaging, a guide wire should be placed initially using the anteroposterior view; then a tunnel in line with the posterior cortex of the femur should be performed using a true lateral view, as described by Schottle et al. $(28,29)$.

A biomechanical study comparing the biomechanical outcomes of two different MPFL reconstruction techniques (the Ochi modified technique with one patellar tunnel and one femoral tunnel and Cerulli's anatomical sail type reconstruction with one curved patellar tunnel and one femoral tunnel) showed that the single-bundle reconstruction technique failed at 171 Newtons $(\mathrm{N})$, with a consequent increased risk of patellar tilt occurrence, while the sail type reconstruction failed at $213 \mathrm{~N}$ with no patellar tilt occurrence. It has been clearly shown that reconstructing the MPFL shape achieves better results because it reduces the influence of technique errors on the functional outcomes (30).

It must always be remembered that MPFL rupture is the result, not the cause, of an altered extensor mechanism; therefore, patellar stabilization with MPFL reconstruction is only the first step to be accomplished.

If other anatomical alterations are encountered, additional/alternative surgical procedures should be considered. In cases in which MPFL rupture occurs as a result of trauma, with no associated anatomical or functional knee alterations, an appropriate rehabilitation program after MPFL reconstruction should be sufficient to achieve a good outcome.

Patellofemoral rehabilitation should address all the dynamic dysfunctions of the leg, such as abnormal leg motion due to proximal impairments (i.e., of the hip) or distal malalignment (i.e., of the foot), because these could influence the dynamic $Q$ angle. A functional rehabilitation program should be preceded by a biomechanical evaluation with quantitative analyses to be used for comparing pre- and post-treatment scores. This evaluation should include muscle strength analysis, joint angle analysis and evaluation of possible muscle imbalance. To ensure the achievement of good and safe results, the clinician could use stabilometric 
analysis, isokinetic tests, EMG and 3D kinematic tests. In conclusion, an acute patellar dislocation in skeletally immature patients should be treated with MPFL reconstruction with physeal sparing; osseous procedures are contraindicated, medial reefing and lateral release are ineffective. Complete biomechanical study of the kinetic chains is crucial in the postoperative management.

\section{References}

1. Vavken P, Wimmer MD, Camathias C, et al. Treating patella instability in skeletally immature patients. Arthroscopy. 2013; 29:1410-1422

2. Mehta VM, Inoue M, Nomura E, et al. An algorithm guiding the evaluation and treatment of acute primary patellar dislocations. Sports Med Arthrosc. 2007;15:78-81.

3. Sanchis-Alfonso V. Background: Patellofemoral malalignment versus tissue homeostasis. In: Sanchis-Alfonso V (Ed) Anterior Knee Pain and Patellar Instability. Springer, Berlin. 2005;3-19.

4. Redziniak DE, Diduch DR, Mihalko WM, et al. Patellar instability. J Bone Joint Surg Am. 2009;91:2264-2275.

5. Nietosvaara Y, Aalto K, Kallio PE. Acute patellar dislocation in children: incidence and associated osteochondral fractures. J Pediatr Orthop. 1994;14:513-515.

6. Stracciolini A, Casciano R, Levey Friedman H, et al. Pediatric sports injuries: a comparison of males versus females. Am J Sports Med. 2014;42:965-972.

7. Lewallen L, McIntosh A, Dahm D. First-time patellofemoral dislocation: risk factors for recurrent instability. J Knee Surg. 2015;28:303-309.

8. Wiberg G. Roentgenographic and anatomic studies on the patellofemoral joint with special reference to chondromalacia patellae. Acta Orthop Scand. 1941;21:319-410.

9. Panni AS, Cerciello S, Maffulli N, et al. Patellar shape can be a predisposing factor in patellar instability. Knee Surg Sports Traumatol Arthrosc. 2011;19:663-670.

10. Senavongse W, Farahmand F, Jones J, et al. Quantitative measurement of patellofemoral joint stability: force-displacement behavior of the human patella in vitro. J Orthop Res. 2003; 21:780-786.

11. Shih YF, Bull AM, Amis AA. The cartilaginous and osseous geometry of the femoral trochlear groove. Knee Surg Sports Traumatol Arthrosc. 2004;12:300-306.

12. Dejour H, Walch G, Neyret P, et al. Dysplasia of the femoral trochlea. Rev Chir Orthop Reparatrice Appar Mot. 1990;76: 45-54.

13. Eckhoff DG, Winter WG. Femoral and tibial torsion. Clin Orthop Relat Res. 1994;(302):2-3.

14. Lee TQ, Anzel SH, Bennett KA, et al. The influence of fixed rotational deformities of the femur on the patellofemoral contact pressures in human cadaver knees. Clin Orthop Relat Res. 1994;(302):69-74.

15. Tennant S, Williams A, Vedi V, et al. Patello-femoral tracking in the weight-bearing knee: a study of asymptomatic volunteers utilising dynamic magnetic resonance imaging: a preliminary report. Knee Surg Sports Traumatol Arthrosc. 2001; 9:155-162.

16. Powers CM. The influence of altered lower-extremity kinematics on patellofemoral joint dysfunction: a theoretical perspective. J Orthop Sports Phys Ther. 2003;33:639-646.

17. Dejour H, Walch G, Deschamps G, et al. Arthrosis of the knee in chronic anterior laxity. Rev Chir Orthop Reparatrice Appar Mot. 1987;73:157-170.

18. Arendt EA, Dejour D. Patella instability: building bridges across the ocean a historic review. Knee Surg Sports Traumatol Arthrosc. 2013;21:279-293.

19. Stefancin JJ, Parker RD. First time traumatic patellar dislocation: a systematic review. Clin Orthop Relat Res. 2007;455:93101.

20. Panni AS, Vasso M, Cerciello S. Acute patellar dislocation. What to do? Knee Surg Sports Traumatol Arthrosc. 2013; 21:275-278.

21. Erickson BJ, Mascarenhas R, Sayegh ET, et al. Does operative treatment of first-time patellar dislocations lead to increased patellofemoral stability? A systematic review of overlapping meta-analyses. Arthroscopy. 2015;31:1207-1215.

22. Smith TO, Donell S, Song F, et al. Surgical versus non-surgical interventions for treating patellar dislocation. Cochrane Database Syst Rev. 2015;2:CD008106.

23. Sillanpää PJ, Mattila VM, Mäenpää $H$, et al. Treatment with and without initial stabilizing surgery for primary traumatic patellar dislocation. J Bone Joint Surg Am. 2009;91:263-273.

24. Colvin AC, West RV. Patellar instability. J Bone Joint Surg Am. 2008;90:2751-2762.

25. Amis AA, Firer P, Mountney J, et al. Anatomy and biomechanics of the medial patellofemoral ligament. Knee. 2003; 10: 215-220.

26. Shah JN, Howard JS, Flanigan DC, et al. A systematic review of complications and failures associated with medial patellofemoral ligament reconstruction for recurrent patellar dislocation. Am J Sports Med. 2012;40:1916-1923.

27. Vavken P, Wimmer MD, Camathias C, et al. Treating patella instability in skeletally immature patients. Arthroscopy. 2013;29:1410-1422.

28. Nelitz M, Dreyhaupt J, Reichel H, et al. Anatomic reconstruction of the medial patellofemoral ligament in children and adolescents with open growth plates: surgical technique and clinical outcome. Am J Sports Med. 2013;41:58-63.

29. Ladenhauf HN, Berkes MB, Green DW. Medial patellofemoral ligament reconstruction using hamstring autograft in children and adolescents. Arthrosc Tech. 2013;26:e151-e154.

30. Placella G, Criscenti G, Tei MM, et al. Reconstruction of the medial patellofemoral ligament: a biomechanical comparison of two techniques. J Orthopaed Traumatol. 2013;14 (Suppl 1):S74. 